\title{
Reseña del libro La educación y los retos de 2018: una visión académica, coordinado por Hugo Casanova Cardiel ${ }^{1}$
}

José-Ángel Beristain-Cardoso

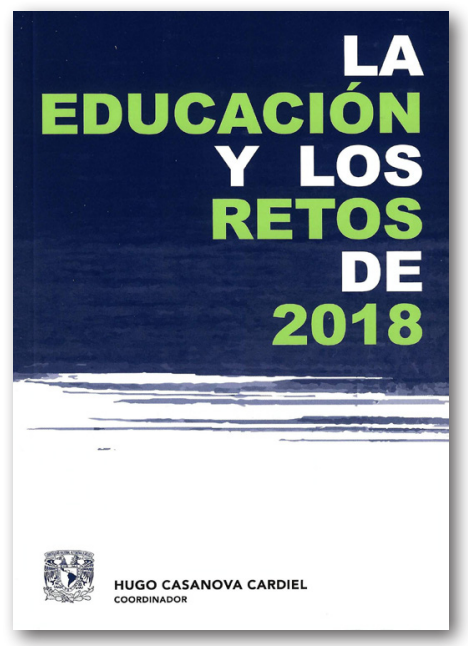

ctualmente se debate en las cámaras y en distintos foros las "puntualizaciones finales de la Reforma Educativa" dadas a conocer por la Secretaría de Educación Pública (SEP), como parte de la configuración de una política educativa del recién gobierno de izquierda en México. Precisamente en este momento adquiere mayor pertinencia esta obra, en primer lugar porque coloca a la "educación" en el centro de la discusión sobre el futuro de la sociedad; y en segundo, porque presenta una reflexión crítica de los investigadores académicos, aquellos que las autoridades educativas no escucharon bien, o tal vez no con la misma atención que a otras voces u opiniones en su momento.

José-Ángel Beristáin-Cardoso

jose_angelb@hotmail.com Mexicano. Doctor en Historia y Etnohistoria, Escuela Nacional de Antropología e Historia (ENAH), México. Estancia posdoctoral (2018-2019), "UNAM. Programa de Becas Posdoctorales", becario del Instituto de Investigaciones sobre la Universidad y la Educación, UNAM, México. Temas de investigación: historia social y cultural del patrimonio, instituciones y políticas culturales en México siglos XX-XXI, vínculo educación-cultura.

\footnotetext{
${ }^{1}$ Hugo Casanova Cardiel (coord.) (2018), La educación y los retos de 2018, México, UNAM.
} 
Este libro, coordinado por Hugo Casanova Cardiel, uno de los autores con una prestigiosa trayectoria en la investigación de politicas universitarias y política educativa en nuestro país, tiene como raíz los encuentros de reflexión de los foros universitarios: "La UNAM y los desafíos de la Nación", los cuáles se realizaron en 2017, con el propósito de aprovechar el conocimiento de los participantes y lograr generar alternativas y posibles respuestas al momento coyuntural en los grandes temas nacionales, entre éstos la cultura y educación. Casanova Cardiel coordina los puntos de vista, reflexiones y propuestas de 10 destacados investigadores académicos de distintas instituciones, partiendo de una "pregunta central": ¿Por qué es importante pensar la educación? La respuesta tiene muchas aristas, y es aquí donde radica la mayor aportación de esta obra, en donde el lector podrá dilucidar y hacer un balance a través de las reflexiones vertidas y con lo propio investigado.

En el texto introductorio "Pensar la educación hoy", de Casanova Cardiel, se redimensiona a la "educación" como "la causa social con mayor consenso de nuestro tiempo" y como "uno de los factores que han contribuido de una manera más notable a la conformación del México actual"; el autor, en primer orden, destaca la capacidad emancipadora de la educación y en segundo, acusa la necesidad del reconocimiento de la creación de lugares orgánicos o instituciones, en donde hombres y mujeres formaron parte de la construcción cultural de nuestro país a lo largo del siglo XX; actores que se fueron abriendo camino desde las misiones culturales, la escuela transformadora, la legitimación de la ciencia y la técnica, la reforma de los textos gratuitos y los intentos de modernización educativa. El legado de la política educativa en nuestro país es desconocido en la más reciente reforma educativa 2013-2017, de tal manera que ésta se tornó duramente "crítica" con el pasado, pero sin bases de consulta, y con un resultado que hoy tiene a los especialistas, docentes, estudiantes y demás actores discutiendo y debatiendo su impacto en las siguientes generaciones. El coordinador de la obra - y este es otro de los principales aciertos - focaliza cinco problemas cruciales de la educación: 1) dimensiones cuantitativas del sistema, 2) reconocimiento de la calidad y sus múltiples criterios implantados, 3) infraestructura y equipamiento, 4) vínculo educación-política y 5) vínculo educación-sociedad (ciudadanía, democracia y seguridad). Entre estos problemas se plantea si los esquemas de gobernanza se deben alejar del discurso para nutrirse de mejores condiciones para una mayor y mejor interactividad social de abajo hacia arriba; y en cuanto a la calidad, se reconocen los acuerdos en cuanto a mejorar instituciones y procesos educativos, pero al mismo tiempo se cuestiona la burocratización de éstos, y más aún si consideramos a la calidad educativa como un conjunto de políticas públicas para la desigualdad, es decir, de una distribución de bienes sociales con base en méritos individuales, que es un tema que cada vez comienza a asumir mayor rigor y profundidad de análisis en recientes investigaciones.

En el libro en conjunto, la mirada plural de los académicos se integra en las siguientes líneas temáticas.

\section{Retos y perspectivas de la educación básica en México}

Ángel Díaz Barriga, investigador emérito del Instituto de Investigaciones sobre la Universidad y la Educación (IISUE) de la Universidad Nacional Autónoma de México (UNAM), en "La Reforma educativa 2013-2017: entre lo fallido y la búsqueda de un contenido pedagógico", logra esclarecer al lector el "revés de la reforma" o la "reforma al revés", es decir, los puntos críticos de una reforma que inicia regulando las relaciones laborales entre el Estado y los profesores, para después definir un proyecto curricular-didáctico que funcionará hasta el final del periodo sexenal del gobierno en turno que la implementó. Con la objetividad y prestigio de su reconocida trayectoria, el autor devela las "tareas pendientes" de una contradictoria reforma educativa que primero 
descalifica socialmente al docente para después buscar su profesionalización, respondiendo más a una lógica de la pedagogía eficientista que a otros factores que también influyen en los resultados del aprendizaje.

Rosa María Torres Hernández, profesora e investigadora de la Universidad Pedagógica Nacional (UPN), en "La educación básica en México en el horizonte de justicia y los derechos", encuentra en la década de los noventa del siglo XX el desarrollo de una fuerte "conciencia" en los actores del proceso pedagógico, para impulsar una verdadera transformación en los sistemas educativos. En esta década se tendió un puente entre la priorización de la universalización y la cobertura de la educación, así como la instalación de una agenda de los derechos y políticas de inclusión social y cultural. En este reconocimiento de los derechos de las poblaciones excluidas se hicieron más visibles las condiciones de vulnerabilidad y discriminación que genera la desigualdad. Partiendo de esta problemática, en este tema se hace una breve reflexión sobre los campos de batalla decisivos que abonan a la justicia social en los territorios de la educación básica.

María de Ibarrola, investigadora del Departamento de Investigaciones Educativas (DIE) del Centro de Investigaciones Avanzadas (Cinvestav) del Instituto Politécnico Nacional (IPN), en "Los retos que plantean 25 años de reformas educativas en México: 1992-2017", propone un interesante ejercicio de la escolaridad que hipotéticamente podrían recibir dos generaciones: 1988 y 2000, partiendo de políticas generales y estadísticas nacionales. Mas allá de la precisa descripción de las particularidades de cada generación, vitales para el contraste de los alcances de la política educativa, y una mejor comprensión del panorama de la Reforma de 2013; la autora realiza un interesante balance de los desafíos de la política educativa en este periodo, señalando al 2018 como el año crítico para la consolidación o abandono sexenal de los proyectos educativos.

\section{Los grandes retos de la educación superior y media superior}

Adrián Acosta Silva, investigador de la Universidad de Guadalajara, en "El gobierno de la educación superior como problema y como desafio", parte del supuesto de la inexistencia de un sistema nacional de educación superior en México, en un contexto en donde los esfuerzos aislados de las instituciones dependen siempre de un marco público, en una especie de "conglomerado" poco articulado, en expansión y crecimiento, y con múltiples instancias de coordinación que gobiernan los pocos núcleos de éste. El lector podrá vislumbrar en esta interesante propuesta un diagnóstico en los últimos 30 años de los efectos económicos, políticos y sociales de las transformaciones en la educación superior, así como la compleja coexistencia - uno de los grandes aciertos en el tema - entre instituciones múltiples y heterogéneas que forman parte de este "conglomerado".

Romualdo López Zárate, investigador de la Universidad Autónoma Metropolitana (UAM-Azcapotzalco), Departamento de Sociología, en "El liderazgo de los rectores, ¿mito o realidad?", coloca a las universidades públicas y autónomas como las depositarias de la confianza y la credibilidad de la sociedad ante la creciente desconfianza hacia las acciones del gobierno. El autor, a través de estudios de caso, devela la ausencia o presencia del liderazgo de los rectores, como una construcción social y cultural, es decir sustentada en la percepción de los sujetos. En esta reflexión el lector podrá identificar a la "legitimidad" como parte vital del liderazgo, la cual sigue teniendo una fuerte esencia del enfoque weberiano, en donde la "dominación" siempre procura fomentar y despertar la creencia en ésta. El papel de la rectoría como empresa social más que de un interés personal, está muy bien sustentado, a través de un adecuado análisis de la trayectoria de los rectores, las principales características de su liderazgo y los tipos de su ejercicio. El lector encontrará distintos elementos que le permitirán realizar un balance en 
el caso de las universidades públicas mexicanas que, como bien señala el autor "juegan hoy el papel de la reserva moral del país".

Humberto Muñoz García, investigador emérito del Instituto de Investigaciones Sociales (IIS) de la UNAM, en "Ideas para discutir la transformación de la universidad pública en México", propone cavilar el ámbito educativo como un escenario donde se disputa la nación, en otras palabras, como una salida de la crisis moral en la que ésta se encuentra. El autor sustenta su propuesta en una crítica muy aguda, y probablemente certera, del contexto social y los desafios de la educación superior, principalmente en los alcances y obstáculos de las políticas oficiales en el desarrollo de las instituciones académicas. Considerando que nuestro país debe transitar a una sociedad del conocimiento, y para lo cual debe adquirir nuevos atributos para adaptarse a los cambios en el contexto, queda en el lector coincidir o discernir sobre los comentarios del autor en relación con las metas logradas en la cobertura, calidad, innovación y vinculación del régimen sexenal en turno.

\section{Los temas críticos de la educación en México: evaluación, financiamiento y equidad}

Javier Mendoza Rojas, investigador del IISUEUNAM, en "El financiamiento de la educación superior: problemas y retos", y como especialista en los procesos de cabildeo legislativo para el presupuesto federal de la educación (2001-2006), comparte sus reflexiones en torno a las universidades públicas agrupadas en la Asociación Nacional de Universidades e Instituciones de Educación Superior (ANUIES); partiendo de las interrogantes sobre los recursos destinados a la educación, los modelos y problemas de financiamiento. El autor hace una efectiva revisión de los "modelos de asignación" y los criterios utilizados para el presupuesto de las universidades públicas en las últimas dos décadas. El lector podrá visibilizar las variaciones y ajustes presupuestales en el gasto de las universidades públicas, el subsidio ordinario de las estatales y los fondos extraordinarios dirigidos a subsistemas, que abarcan desde instituciones politécnicas hasta escuelas normales. Otra de las virtudes de este texto es la identificación de los factores internos y externos que influyen en el presupuesto educativo, así como las disociaciones entre el incremento del financiamiento y el crecimiento de la matrícula en un esquema de financiamiento que muestra síntomas de agotamiento.

Mario Rueda Beltrán, investigador del IISUEUNAM, en "La evaluación educativa: límites y desafios", con una minuciosa y sistémica reflexión propone cómo mejorar el objeto evaluado, como finalidad central de la evaluación, y como una manera de un mejor aprovechamiento de los resultados de todas las acciones emprendidas. El autor siempre ha hecho énfasis en sus investigaciones sobre la ausencia del pasado en el nuevo modelo educativo, y la importancia de revivir experiencias educativas para la reconstrucción de la política educativa. En un análisis y recuento de la presencia de la evaluación educativa en los últimos 30 años, el lector podrá contrastar entre las diferentes estrategias dirigidas a la educación obligatoria y aquellas orientadas a la educación superior, así como visibilizar la complejidad del sistema educativo mexicano y la inviabilidad de contar con una sola estrategia de evaluación.

Imanol Ordorika, investigador del Instituto de Investigaciones Económicas (IIE) de la UNAM, en "Política, educación y política", sugiere una visión aguda y crítica sobre el orden político de la educación, tomando como punto de partida la historia. El autor identifica concisamente niveles álgidos de discusión y debate pedagógico en diferentes momentos históricos, entre éstos la construcción de la nación en el siglo XIX y XX; no olvidemos mencionar al estado de Veracruz como cuna de reformas educativas, aquellas emprendidas por el maestro Carlos A. Carrillo y la introducción de la "Enseñanza objetiva" (1885), las "Lecciones de cosas" de Enrique 
Laubscher (1881), y la creación de la Escuela Normal de Profesores en Jalapa por Rébsamen (1887), entre otras acciones que desaprobaban la inflexibilidad del sistema educativo mexicano y sus métodos. El lector contará con distintos ingredientes para discernir que el gran debate nacional en el sexenio que termina no sucedió en elámbito de los recursos naturales como el petróleo, sino en el de la educación, y todo, como enfatiza claramente el autor, para resolver un problema meramente político.

Roberto Rodríguez, investigador del Instituto de Investigaciones Sociales (IIS) de la UNAM, en "La equidad educativa desde la perspectiva del derecho a la educación", parte del desentrañamiento entre derecho educativo y el derecho a la educación que se inserta en el ámbito de los derechos humanos, en un contexto de un México que imparte una educación injusta, es decir, con normas que no se cumplen en la realidad. El autor sugiere no desestimar el problema de la calidad de los datos estadísticos de la cobertura educativa, y así, en esta especie de enredo entre educación de mala calidad y pobreza, cabe discutir y debatir el cómo hacer llegar calidad educativa en donde no la hay, que es precisamente en las zonas rurales, urbanas marginadas y en las comunidades indígenas.

Hoy en día el futuro de la "educación" en México está nuevamente en las cámaras legislativas, en momentos de gran tensión, entre "reformar lo reformado" o "abrogar lo reformado"; y es aquí dónde "2018” se erige nuevamente como el "año crítico", y en dónde las aportaciones y visiones en los foros de debate y discusión de los investigadores académicos pueden ser de vital importancia, principalmente para aprovechar las diversas experiencias educativas. Las propuestas en esta obra, sin lugar a duda, enriquecen la reflexión colectiva, y propician la difusión de espacios de debate, que más allá de quedarse en las memorias de los foros, invite al análisis inter y multidisciplinario de los temas trascendentales de la nación. El coordinador de esta obra está muy consciente de las vertientes que genera para los lectores, que pueden ser profesores, estudiantes, sociólogos, historiadores de la educación, pedagogos, representantes de organismos gubernamentales o no gubernamentales, entre otros, es decir, todos aquellos actores involucrados en el campo educativo. Casanova Cardiel reconoce que esta obra sólo aborda algunos ángulos del marco educativo, lo cual es completamente comprensible, y sin duda deja la puerta abierta para una segunda parte de reflexiones y aportaciones, en donde se puedan incluir temas sobre educación preescolar, media superior, posgrado y modalidades como la educación artística, en un contexto en donde recién se rompió el binomio educación-cultura sellado desde el vasconcelismo; la educación especial en sus distintas perspectivas y problemas de inclusión, la educación física centrada en el enfoque orgánico-deportivo dentro del contexto de la globalización, y la educación intercultural como proyecto social más allá del concepto.

\section{Cómo citar este artículo:}

Beristáin-Cardoso, José-Ángel (2019), "Reseña del libro La educación y los retos de 2018: una visión académica, coordinado por Hugo Casanova Cardiel," en Revista Iberoamericana de Educación Superior (RIES), México, UNAM-IISUE/Universia, vol. X, Núm. 28, pp. 208212, DOI: https://doi.org/10.22201/iisue.20072872e.2019.28.437 [consulta: fecha de última consulta]. 